\title{
Astronomy with Radioactivities
}

\author{
Roland Diehl $^{\mathrm{A}, \mathrm{C}}$ and Maria Lugaro ${ }^{\mathrm{B}}$ \\ ${ }^{A}$ Max-Planck-Institut für extraterrestrische Physik, D-85741 Garching, Germany \\ ${ }^{B}$ Monash Centre for Astrophysics, School of Mathematical Sciences, Monash University, \\ Melbourne, Vic. 3800, Australia \\ ${ }^{\mathrm{C}}$ Corresponding author. Email: rod@mpe.mpg.de
}

Cosmic radioactivity represents a cross-disciplinary theme and an interesting alternate viewpoint on cosmic nuclear astrophysics. Radioactive isotopes and their decay provide unique messages from sites of cosmic nucleosynthesis, as the decay is mediated by weak interaction physics and independent of environmental conditions. The radioactive clock of various isotopes traces stellar mixing processes and the process of solidification of bodies when the solar system was formed. Isotopic abundances directly reflect the conditions of their formation in the nucleosynthesis site, which is unobservable otherwise. Measurements range from meteorites and their included stardust grain compositions through to cosmic rays and electromagnetic radiation from infrared to gamma ray wavelengths. Thus, various astronomical disciplines with their different messengers of cosmic nucleosynthesis as seen through unstable, decaying isotopes are linked to the physics of nuclear reactions, and to theories and models of the variety of cosmic nucleosynthesis sites and of cosmic isotopic evolution.

In this issue, several papers illustrate the power of this view ('Lecture notes' have also been compiled with many details; see Introduction to Astronomy with Radioactivities by R. Diehl, D. H. Hartmann, and N. Prantzos, Springer LNP 812, 2010). These PASA papers derive from a scientific gathering in March 2011 on Phillip Island, Victoria, Australia - the 7th Astronomy with Radioactivities workshop in a series which started in 1996 and is held every two years. Once again, it brought together astronomers observing electromagnetic radiation from various different bands as well as cosmic rays, experimentalists working on meteoritic samples, nuclear physics experimenters, and a diversity of theoretical astrophysicists working on star formation, stellar evolution, supernova explosions and their neutron star remnants, accretion flows, cosmic rays, and cosmic dust formation. This rare assembly of such diverse expertise is characteristic of these workshops, and stimulates crossdisciplinary insights and fertilization. We now briefly summarise the workshop's findings.

Paradigm changes on how stars are believed to form from interstellar gas were illustrated by Matthew Bate: gas flows, angular momentum transport, and stellar collisions - all in a highly dynamic, and in parts chaotic, interplay - together convert a few percent of the gas in an interstellar cloud into stars. Born typically in groups and clusters, a significant fraction of those stars leave their parental site and companions as a result of collisions and interactions. Hence arises the diversity of bound and open star clusters, moving groups, and seemingly isolated stars. Kurt Liffman showed how angular momentum transport near a newly formed star incurs a convective zone between the inner accretion disk surrounding the star and its dipole magnetic field, a region where outflows and jets are believed to form. Details of such accretion environments determine the condensation of solid bodies from this prestellar material, such as refractory metal nuggets that are believed to be carriers of star-forming material in its pristine composition.

Understanding condensation sequences and chemical or physical reprocessing is a prerequisite to disentangling the properties of the presolar nebula from meteoritic inclusions. Many such meteoritic inclusions have been recognised to be extra-solar stardust from their dramatic deviations in isotopic signatures with respect to solar system material. Remarkable precision has been achieved in determining isotopic composition together with spatial/morphological detail. It had been easiest to separate the hard and dense silicon carbide grains, but recent advances also make oxide grains accessible to such studies, significantly broadening the range of conclusions due to their different condensation conditions. Uli Ott (Ott et al. 2012) showed the potential held by frontier instrumentation which will be able to determine isotopic information even in nanodiamonds, particles which include only about a thousand atoms each. A concerted effort of chemical separation with accelerator mass spectroscopy and secondary ion mass spectrometry may now also address $r$-process details. Because AGB stars are copious and efficient dust producers, their nucleosynthesis patterns are clearly seen in such stardust studies, and have been exploited to constrain the dynamics of the stellar structure adjustments in these giant stars, where two concentric shells undergo nuclear burning. Various aspects of stardust isotopic results with respect to the $s$-process and nuclear reaction rates were discussed by Maria Lugaro, Amanda Karakas, Sara Palmerini, and Joelene Buntain. We also know that supernovae produce dust, and Don Clayton showed here that the chemistry is out of equilibrium in the rapidly expanding ejecta, and thus more difficult to understand in its solid-particle condensation aspects. Nevertheless, 
meteoritic inclusions and their isotopic ratios as revealed in terrestrial laboratories can be seen as global astronomical telescopes for the study of stellar structure and nucleosynthesis.

Jenny Feige (Feige et al. 2012) discussed the fascinating opportunities from radioactive ${ }^{60} \mathrm{Fe}$ isotopes found on Earth in deep-sea ocean crust material. This is an alternative to meteoritic studies in analysing supernova ejecta material directly, and Jenny discussed how such ejecta may propagate from a supernova to the Earth and into the ocean crust, from where it has been measured through accelerator mass spectroscopy.

Anton Wallner (Wallner et al. 2012) presented an educational overview on the capabilities of the accelerator mass spectroscopy technique for various purposes, such as analysing the above-mentioned small ocean crust material sample, and he also addressed its ability to measure nuclear reaction cross-sections with very low values in particular. Robert Tribble showed how nuclear physics experiments have advanced to generate radioactive ion beams for the measurement of nuclear reactions further away from the valley of stable isotopes, such as those relevant to cosmic environments. Robert discussed the different approaches to measure the variety of reaction channels, emphasising indirect methods such as the asymptotic normalization coefficient approach, which avoids nuclear model complexities to better address reaction rates at low interaction energies typical for stars and novae.

Tim Beers presented the prospects for nucleosynthesis data from astronomical telescopes. Digital sky surveys are currently in progress and will allow a staged collection of data for stars at the metal-poor end. High-resolution spectra will enable elemental and partly isotopic composition studies of the thousands of stars that are assumed, from their low content in elements heavier than helium, to be among the first to have formed in our Galaxy. Tim showed that binaries must have been at the origins of the subset of metal-poor stars which show a striking richness in carbon and thus must have been seeded with peculiar enrichment in carbon very efficiently. Several authors discussed the implication of binaries where a giant star develops more rapidly and leaves behind a carbon-rich but otherwise extremely iron-poor star as we see it today. Sara Bisterzo showed what has been learned about the $s$-process in the early Galaxy from these metal-poor stars. Richard Stancliffe pointed out that among the C-enriched EMP stars, the simultaneous signatures of n-capture elements from the $s$ - and $r$-process are confusing, and cannot be understood from presently discussed scenarios. This example illustrates the various observational biases that are often overlooked: apparently simple chemical enrichment patterns may actually be superpositions of a variety of enrichment paths of different stellar populations. This will be important in extracting information from $r$-process elements $\mathrm{Th}$ and $\mathrm{U}$ which are used as cosmochronometers. Clearly, we need to exploit different ways to measure cosmic abundances not just in stars, but also in interstellar matter.
Several contributions discussed astronomical measurements of radioactive decays and positron annihilation, which are rather direct ways to observe isotopes as they decay in interstellar space. Mark Leising highlighted what such measurements may tell us about supernova explosion mechanisms and supernova expansion, thus complementing the more precise but model-dependent UVOIR spectra and light curves from supernovae and their interpretations. Roland Diehl showed that gamma ray measurements from long-lived isotopes can address the poorly known epoch between supernovae and their bright remnants and dispersal of nucleosynthesis ejecta into the cold component of the interstellar gas from which next-generation stars may form. Richard Rothschild discussed positron annihilation gamma ray measurements, and he emphasised that well known positron sources and their distribution in the Galaxy can explain those observations from Galactic structure - a view debated by other scientists due to the large contrast between Galactic disk and bulge. Matthew Baring showed that supernova remnants may still be rightly considered as the major cosmic ray accelerators, but substantial unknowns in the shock acceleration details, and also inconsistencies with respect to cosmic ray spectra and composition over energy clearly show that the astrophysics of relativistic particle acceleration is far from being understood.

Nina Tetzlaff (Tetzlaff et al. 2012) showed yet another approach to investigating nucleosynthesis sources across our Galaxy through detailed analysis of the locations of their remnant neutron stars, and back-propagating these towards currently observed massive star cluster locations.

Theorists study how different cosmic sites may be able to contribute to cosmic isotopic abundances and nucleosynthesis. Davis Chamulak explained the issues with modelling thermonuclear supernovae (type Ia) and their potential uncertainties with respect to metallicity dependence, an important aspect of their use as cosmological distance indicators. Ko Nakamura and Tak Hayakawa discussed core-collapse nuclear reactions related to the copious neutrinos flux when a protoneutron star is formed in these events. Marco La Cognata discussed how the particular isotope ${ }^{8} \mathrm{Li}$ can tell us more about core-collapse supernova interiors, and also about nucleosynthesis in the Big Bang. Duncan Galloway finally showed that nuclear reactions on surfaces of neutron stars may also provide interesting characteristic signatures which can be exploited to understand the structure of neutron stars.

This workshop once again set the stage for crossdisciplinary discussions of astronomical, experimental, and theoretical approaches to astrophysics related to radioactive isotopes. Participants valued the setting and program of the workshop, where basic questions could be posed and discussed in more depth than what is possible on larger-scale conferences. We are grateful for the support of the Victorian Endowment for Science, Knowledge, and Innovation, and Monash University. Many participants left the beautiful (but unusually cold) island south of Melbourne with new stimulus for their own 
research. They will be looking forward to the next workshop on Astronomy with Radioactivities in due course, as well as other events such as 'Nuclei in the Cosmos XII' in Cairns, Australia, in August 2012.

\section{References}

Feige, J., Wallner, A., Winkler, S. R., Merchel, S., Fifield, L. K., Korschinek, G., Rugel, G. \& Breitschwerdt, D., 2012, PASA, 29, 109
Ott, U., Besmehn, A., Farouqi, K., Hallmann, O., Hoppe, P. Kratz, K.-L., Melber, K. \& Wallner, A., 2012, PASA, 29, 90 Tetzlaff, N., Schmidt, J. G., Hohle, M. M. \& Neuhäuser, R., 2012, PASA, 29, 98

Wallner, A., et al., 2012, PASA, 29, 115 